\title{
BMJ Open A hospital-based observational cohort study exploring pain and biomarkers in patients with hand osteoarthritis in Norway: The Nor-Hand protocol
}

\author{
Marthe Gløersen, ${ }^{1}$ Elisabeth Mulrooney, ${ }^{1}$ Alexander Mathiessen, ${ }^{1}$ \\ Hilde Berner Hammer, ${ }^{1}$ Barbara Slatkowsky-Christensen, ${ }^{1}$ Karwan Faraj, ${ }^{2}$ \\ Thore Isaksen, ${ }^{3}$ Tuhina Neogi, ${ }^{4}$ Tore K Kvien, ${ }^{1,5}$ Karin Magnusson, ${ }^{6}$ \\ Ida Kristin Haugen ${ }^{1}$
}

To cite: Gløersen M, Mulrooney E, Mathiessen A, et al. A hospital-based observational cohort study exploring pain and biomarkers in patients with hand osteoarthritis in Norway: The Nor-Hand protocol. BMJ Open 2017;7:e016938. doi:10.1136/ bmjopen-2017-016938

- Prepublication history for this paper is available online. To view these files please visit the journal online (http://dx.doi. org/10.1136/bmjopen-2017016938).

Received 27 March 2017 Revised 1 June 2017

Accepted 20 July 2017

\section{CrossMark}

${ }^{1}$ Department of Rheumatology, Diakonhjemmet Hospital, Oslo, Norway

${ }^{2}$ Department of Radiology, Diakonhjemmet Hospital, 0slo, Norway

${ }^{3}$ Department of Radiology, Volvat Medical Center, Oslo, Norway

${ }^{4}$ Clinical Epidemiology Research and Training Unit, Boston University School of Medicine, Boston, USA

${ }^{5}$ The Medical Faculty, Institute for Clinical Medicine, University of Oslo, Olso, Norway

${ }^{6}$ National Advisory Unit on

Rehabilitation in Rheumatology, Diakonhjemmet Hospital, Oslo, Norway

Correspondence to

Dr Ida Kristin Haugen;

haugen_ida@hotmail.com

\section{ABSTRACT}

Introduction We have limited knowledge about the underlying disease mechanisms and causes of pain in hand osteoarthritis $(\mathrm{OA})$. Consequently, no diseasemodifying drug exists, and more knowledge about the pathogenesis of hand $\mathrm{OA}$ is needed, as well as a validation of different outcome measures. Our first aim of this study is to explore the validity of various imaging modalities for the assessment of hand OA. Second, we want to gain a better understanding of the disease processes, with a special focus on pain mechanisms.

Methods and analysis The Nor-Hand study is a hospitalbased observational study including 300 patients with evidence of hand $\mathrm{OA}$ by ultrasound and/or clinical examination. The baseline examination consists of functional tests and joint assessment of the hands, medical assessment, pain sensitisation tests, ultrasound (hands, acromioclavicular joint, hips, knees and feet), CT and MRI of the dominant hand, conventional radiographs of the hands and feet, fluorescence optical imaging of the hands, collection of blood and urine samples as well as self-reported demographic factors and OA-related questionnaires. Two follow-up examinations are planned. Cross-sectional analyses will be used to investigate agreements and associations between different relevant measures at the baseline examination, whereas the longitudinal data will be used for evaluation of predictors for clinical outcomes.

Ethics and dissemination The protocol is approved by the Norwegian Regional Committee for Medical and Health Research Ethics (Ref. no: 2014/2057). The participants receive oral and written information about the project and sign a consent form before participation. They can, whenever they want, withdraw from the study, and all deidentified data will be safely stored on the research server at Diakonhjemmet Hospital. Results will be presented at international and national congresses and in peerreviewed rheumatology journals. Trial registration number NCT03083548; Pre-results.

\section{INTRODUCTION}

\section{Osteoarthritis}

Osteoarthritis (OA) is the most common rheumatic joint disease in industrialised countries,

\section{Strengths and limitations of this study}

- To the best of our knowledge, the Nor-Hand study is the first large-scale hand osteoarthritis cohort $(n=300)$ with such a broad evaluation of pain, including patient-reported questionnaires and pain sensitisation tests, which will lead to increased knowledge about pain mechanisms and pain outcomes.

- Due to a thorough examination of structural and inflammatory osteoarthritis by multiple imaging modalities, we will be able to validate and compare different imaging outcome measures.

- Blood and urine are stored in a biobank, allowing us to study soluble biomarkers.

- Follow-up examinations are planned, giving us the opportunity to study predictors for future disease outcomes.

- The Nor-Hand study is limited by inclusion of mainly women, restricting the analyses comparing men and women, as well as the recruitment of patients from specialist care only, limiting the generalisability to patients in primary care.

and is increasing in prevalence due to higher obesity rates and an ageing population. In 2008, it was estimated that nearly 27 million people in the USA had OA. ${ }^{1}$ Any joint in the body can be affected, but $\mathrm{OA}$ is most prevalent in the weight-bearing joints, such as the knees and hips, as well as in the spine and the hands. Today, we know that the whole joint is affected by the disease, but more research is needed to gain a better understanding of the pathogenesis. Limited research has been performed on hand OA, despite being a prevalent disorder that causes pain, fatigue, functional limitations and reduced health-related quality of life. $^{2}$ 
Hand OA is most common in the interphalangeal and thumb base joints. The prevalence increases with age, and radiographic signs of hand $\mathrm{OA}$ are found in the majority of elderly people. ${ }^{3}$ However, hand OA is not only a frequent disease among the elderly, but also a common cause of disability and pain in the middle-aged population. Data collected in the population-based Framingham study estimated that the prevalence of symptomatic hand OA was $14 \%$ among women and $7 \%$ among men between 40 and 84 years of age. ${ }^{4}$ Different subsets of hand OA have been proposed, such as non-erosive versus erosive, and interphalangeal versus thumb base OA. The pathogenesis, risk factors, epidemiology and impact on daily activity vary across the subsets. ${ }^{56}$ More research is needed to gain a better understanding of this highly heterogeneous and frequent disorder. ${ }^{3}$

\section{Imaging modalities}

Hand $\mathrm{OA}$ is mainly a clinical diagnosis. However, conventional radiographs are commonly used if there is doubt about the clinical diagnosis. Typical radiographic features, such as osteophytes, joint space narrowing, subchondral sclerosis and cysts, are used to confirm the diagnosis. ${ }^{7}$ In addition, radiographic central erosions are found in patients with erosive hand OA. European League Against Rheumatism (EULAR) evidence-based recommendations suggest that further imaging investigation is rarely needed for the diagnosis of hand $\mathrm{OA}^{7}$ However, modern imaging techniques play an important role in OA research. Radiographs are only able to identify changes of the bone, and will only indirectly show loss of cartilage. Other imaging methods, such as ultrasound and MRI, can visualise, for example, soft tissue abnormalities including synovitis, and more research is needed in order to explore the role of other imaging modalities in OA research and clinical trials. ${ }^{78}$

Ultrasound appears beneficial compared with conventional radiography because it allows a multiplanar and more dynamic evaluation of both structural and inflammatory OA features without ionising radiation. The disadvantage of ultrasound is that the scoring is operator dependent, related to interpretation of findings and the actual performance of the examination. Both a scoring system with definitions of osteophytes, grey-scale synovitis and power Doppler as well as an atlas with examples of osteophytes have been developed, which may increase the reliability of the scoring. ${ }^{910}$

MRI provides a multiplanar visualisation, and has a unique advantage in detecting multiple-tissue pathology in joints. ${ }^{11}$ MRI is the only imaging modality that is able to show bone marrow lesions (BMLs). Scoring systems for evaluation of MRI features in both interphalangeal OA and thumb base OA have been developed, but further research is needed to validate these scoring systems. ${ }^{12-14}$

Both MRI and ultrasound may be more sensitive than conventional radiographs in detecting early structural changes in hand $\mathrm{OA},{ }^{915}$ possibly because they permit a multiplanar visualisation of the joints. However, MRI is not more sensitive than conventional radiographs in detecting structural progression over 5 years. ${ }^{16}$ Synovitis and BMLs are associated with joint tenderness, and predicts future structural progression. ${ }^{17-22}$ These studies indicate that ultrasound and MRI can identify patients who are more likely to have a progressive disease. However, there might be discrepancy between imaging findings and pain, which underlines that the pain experience is subjective and varies among patients. Therefore, pain might not be a good indicator for the severity of the disease. ${ }^{23}$ Furthermore, lowering the severity of synovitis may represent a treatment target, and more research is needed to explore whether treating inflammation leads to less pain and structural progression in patients with hand OA. ${ }^{24}$

Indocyanine green (ICG)-based fluorescence optical imaging (FOI) is a new imaging modality, and studies have indicated that this can be used to detect synovitis in systemic inflammatory joint diseases. ${ }^{25} 26$ However, previous results are conflicting, as another study has shown lower sensitivity of FOI for detecting joint inflammation as compared with MRI. ${ }^{27}$ Only one previous study has explored the performance of FOI in detection of OA-related inflammation. Glimm et al detected active inflammation with both FOI and ultrasound in a small study of patients with hand OA, and underlined that an inflammatory component could be important in the OA disease process. ${ }^{28}$

In a systematic review about imaging techniques, Saltzherr et al concluded that MRI and ultrasound appear to be the most promising imaging modalities in the future detection of hand OA. However, they also conclude that more research is needed. ${ }^{29}$

\section{Pain}

Pain is the primary reason why most patients with OA seek medical help, ${ }^{30}$ and may lead to reduced quality of life and reduced physical function. ${ }^{2}$ The experience of pain varies widely between individuals. Biological, social and psychological factors, such as prior experiences, previous injuries, heredity, current mood, coping strategies and social differences, are considered to be important in the perception of pain. ${ }^{31}$

Only few questionnaires for evaluation of pain have been validated in patients with hand OA. Hence, development and validation of more questionnaires to be used in future research are needed to better understand the complexity of the pain process. In addition to the Visual Analogue Scales (VAS) and Numeric Rating Scales (NRS), the Australian/Canadian (AUSCAN) hand index is the only currently available hand pain questionnaire that has been comprehensively tested and validated, including on Norwegian patients. ${ }^{32}$ The AUSCAN questionnaire is recommended for use in clinical trials to assess three important aspects of hand OA: pain, stiffness and difficulties with daily activities. ${ }^{33} 34$ However, the questionnaire does not evaluate different pain characteristics and assesses only the intensity of pain in different situations, 
and not the pain frequency. It is important to validate and develop additional questionnaires that can be used in clinical trials, as a step towards the development of more targeted therapy in hand OA.

The OA-associated pain has originally been connected to nociceptive pain because of local tissue damage. However, studies in the recent years have indicated that there is abnormal pain sensitivity in OA, which may have implications for pharmacological pain management in these patients. ${ }^{30}$ Local tissue injury and inflammation in OA may cause an increased response to nociceptive stimuli (ie, peripheral sensitisation). In patients with OA, pain and tenderness might also be increased in areas away from the affected joint, suggesting that central modulation of local nociceptive inputs play a role. ${ }^{36}$

Studies of knee OA have shown that neuropathic-like symptoms, such as spontaneous electric shock-like pain or tingling sensations, may be present in patients with OA. Questionnaires like PainDETECT have been developed to identify neuropathic pain, originally in patients with low back pain. ${ }^{37}$ In this study, we use a modified version of the PainDETECT questionnaire to assess possible neuropathic-like pain in the hands, like has been done in previous studies of knee OA. ${ }^{38}$

Previous OA studies on pain sensitisation mechanisms and neuropathic-like pain have mainly been performed on patients with knee OA. Patients with knee OA have lower pressure pain threshold measurements than healthy controls, which suggest the presence of sensitisation mechanisms. ${ }^{39}$ Furthermore, several studies support the hypothesis that sensitisation mechanisms are involved in the development of pain in knee OA. ${ }^{40}$ Only few small studies have been performed in patients with hand OA, and lower pain thresholds in the hand joints have been demonstrated in these patients. ${ }^{42-44}$ In patients with hand OA, functional MRI scans have shown increased activation in areas of the brain associated with central sensitisation during the performance of painful activities, but not in healthy controls. ${ }^{45}$ Hence, more studies with a larger number of patients are important to gain a better understanding of the role of sensitisation and neuropathic pain in hand $\mathrm{OA}$, which may have consequences for the choice of treatment.

\section{Aims of the project}

By using data from the baseline examination of the Nor-Hand study, the main aim of this project is to gain a better understanding of the disease processes, with a special focus on pain, in hand OA. Different phenotypes of hand OA will be explored in order to identify subgroups that may benefit from different treatment strategies. We have included questionnaires that have not been previously used to assess pain in hand OA. These questionnaires will be validated and used to characterise pain phenotypes in hand OA. We want to investigate risk factors for pain in a biopsychosocial framework in order to better understand risk factors for poor patient outcomes, and assess whether peripheral and central sensitisation influence joint pain in hand OA. In addition, we want to compare and validate OA biomarkers, including both newer and more established imaging modalities. As far as we know, the Nor-Hand study is the first large study to use FOI to investigate hand $\mathrm{OA}$, and this imaging method will be validated against MRI, ultrasound and clinical examination. Other imaging techniques such as ultrasound and MRI scoring systems will also be further validated against, for example, patient-reported outcomes and other biomarkers. Blood and urine samples are stored in a biobank. Soluble biomarkers, including, for example, inflammatory markers, may be examined for exploration of disease pathways in hand OA. In future longitudinal analyses, we will use data from the baseline examination to explore risk factors for both symptom-based and imaging-based disease progression as well as the sensitivity to change and the inter-relationship between OA biomarkers and patient-reported outcomes.

\section{METHODS AND ANALYSIS Design and setting}

The Nor-Hand study is a hospital-based observational cohort study. The study is prospective, and follows 300 patients with hand OA over approximately 8 years with three planned examinations. Patients between the ages of 40-70 years are recruited into Nor-Hand via two channels. One channel is through the rheumatology outpatient clinic at Diakonhjemmet, where patients referred to the hospital are screened for eligibility. Patients are asked to participate in the study if the rheumatologist confirms the diagnosis of hand $\mathrm{OA}$ and, at the same time, excludes other differential diagnoses. Patients are similarly recruited to the study through the 'OA school' organised by the Department of Rheumatology at Diakonhjemmet Hospital. The 'OA school' is a 1-day multidisciplinary course, to which patients can be directly referred from their general practitioner. However, only a few patients are recruited through this channel, as most are recruited through the outpatient clinic. Both populations are identically screened according to the eligibility criteria. Two follow-up examinations of the participants are planned. We plan to repeat all investigations from the baseline examination at the follow-up. However, if we do not get the funding to repeat all examinations, we will, at a minimum, include conventional radiographs and patient-reported outcomes.

\section{Patients}

The study population consists of men and women between the ages of 40 and 70 years. However, due to the female predominance among patients with hand OA referred to specialist care, the participants in the study are mainly women. Their diagnosis of hand $\mathrm{OA}$ is proven either by ultrasound and/or clinical examination performed by a rheumatologist at the rheumatology outpatient clinic at Diakonhjemmet Hospital. All rheumatologists at the department are encouraged to recruit patients to the 


\section{Box Inclusion and exclusion criteria}

\section{Inclusion criteria}

- Age between 40 and 70 years at screening

- Proven hand osteoarthritis by clinical examination and/or ultrasound

- (1) Clinical examination criteria: Heberden/Bouchards nodes and/ or bony enlargement, squaring and/or deformity of the thumb base and no clinical signs of inflammatory arthritis (eg, soft tissue swelling of two or less metacarpophalangeal (MCP) joints, and no soft tissue swelling of the wrist). (2) Ultrasound criteria: Osteophytes in the interphalangeal joints and/or the thumb base, and no signs of inflammatory arthritis (eg, synovitis with power Doppler activity in two or less MCP joints and no synovitis with power Doppler activity in the wrist).

- Capable of understanding and signing an informed consent form

- Provided a written informed consent to participate in the study

\section{Exclusion criteria}

- Diagnosis of inflammatory arthritic disease, for example, seropositive or seronegative rheumatoid arthritis, psoriatic arthritis, reactive arthritis, spondyloarthritis or arthritis related to connective tissue disorders (self-reported or from the medical chart)

- Diagnosis of psoriasis (self-reported, from the medical chart or presence of skin lesions suspect of psoriasis)

- Erythrocyte sedimentation rate $>40 \mathrm{~mm} /$ hour and/or $\mathrm{C}$ reactive protein $>20 \mathrm{mg} / \mathrm{L}$, without a known ongoing infection

- Anti-cyclic citrullinated protein and/or rheumatoid factor positivity

- Ferritin $>200 \mu \mathrm{g} / \mathrm{L}$ for women and $>300 \mu \mathrm{g} / \mathrm{L}$ for men and s-iron/stotal iron binding capacity above $50 \%$ to rule out haemochromatosis

- Major comorbidities (eg, severe malignancies, severe diabetes mellitus, severe infections, uncontrollable hypertension, severe cardiovascular disease or severe respiratory disease)

- Mental or psychiatric disorders, alcohol or drug abuse, language difficulties or other factors that make compliance to the study protocol difficult.

study. The rheumatologists can choose whether they examine for OA using clinical examination or ultrasound (or both). The time between screening examination can vary from a few days to several months depending on the patients' availability and the fact that the screening started well ahead of the baseline examination (March 2015). In addition, all patients must be able to sign and understand an informed consent form. The inclusion criteria are summarised in box. At the time of screening, patients are excluded from the study if they meet any of the exclusion criteria (box).

\section{Sample size}

The number of patients included in this study is not determined by power calculations but is a pragmatically chosen number based on experience from previous studies. ${ }^{2} 1846$ Based on available funding, it is found feasible to include 300 participants in the study.

\section{Assessments}

The patients are invited to a test evening, when most of the examinations, with the exception of CT, conventional radiography and MRI, are performed. One afternoon every week, a team of six trained medical students and one rheumatologist at Diakonhjemmet Hospital examine on average eight new patients. Every test evening lasts for 3-4hours, and one medical student performs the medical assessment and the functional tests, another student performs the pain sensitisation tests, a third student performs the FOI, a fourth student performs the ultrasound of the upper extremities, a fifth student performs the ultrasound of the lower extremities, whereas a sixth student collects blood and urine samples for the biobank. Joint assessment is performed by the rheumatologist. The examiners are blinded for the other results. The participants are also asked to respond to questions about demographic and clinical factors, in addition to OA specific questionnaires. CT, conventional radiography and MRI are performed after every test evening. We aim to assess all 300 patients enrolled in the study with all the investigations described in this protocol (except for participants that have contraindications for some of the investigations, as specified under the description of each investigation).

\section{Questionnaires}

Before the test evening, every patient receives an e-mail with a link to an electronic case report form (eCRF). Alternatively, the patient receives the questionnaires in paper form if needed. The eCRF includes demographic questions, questions about lifestyle, use of drugs, previous surgeries and alternative therapies (table 1). In addition, the patient is asked to answer OA-related questionnaires, including questions about health-related quality of life, psychological health, joint pain and physical function (table 2). All these standardised questionnaires are administered in Norwegian.

\section{Medical assessment}

A trained medical student measures the height of the patient to the nearest millimetre in the standing position. The weight of the patient is measured in kilograms with one decimal precision, while the patient is barefoot and with minimal clothing. Hip circumference is measured around the greater trochanters, while waist circumference is measured midway between the lowest rib and the iliac crest, after the patient has taken a deep breath in and out. Measurements are taken to the nearest millimetre. The patient is then asked to rest for $5 \mathrm{~min}$ before the blood pressure is measured in the sitting position. The measurements are repeated until two consecutive systolic and diastolic pressures have a difference of less than $5 \mathrm{~mm} \mathrm{Hg}$. The last measurements of both systolic and diastolic blood pressures are noted. In addition, the heart rate is taken after $5 \mathrm{~min}$ of rest.

The patient is also asked to answer a self-administered comorbidity questionnaire. ${ }^{47}$ This questionnaire was developed by a panel of five physicians who chose 12 groups of the most frequent medical conditions in general practice. The language of the questionnaire is simplified so it can be easily understood, without any prior medical knowledge. Questions about treatment and impact of the conditions on daily activities are included in 
Table 1 Questions related to demographic factors and clinical history in the case report form

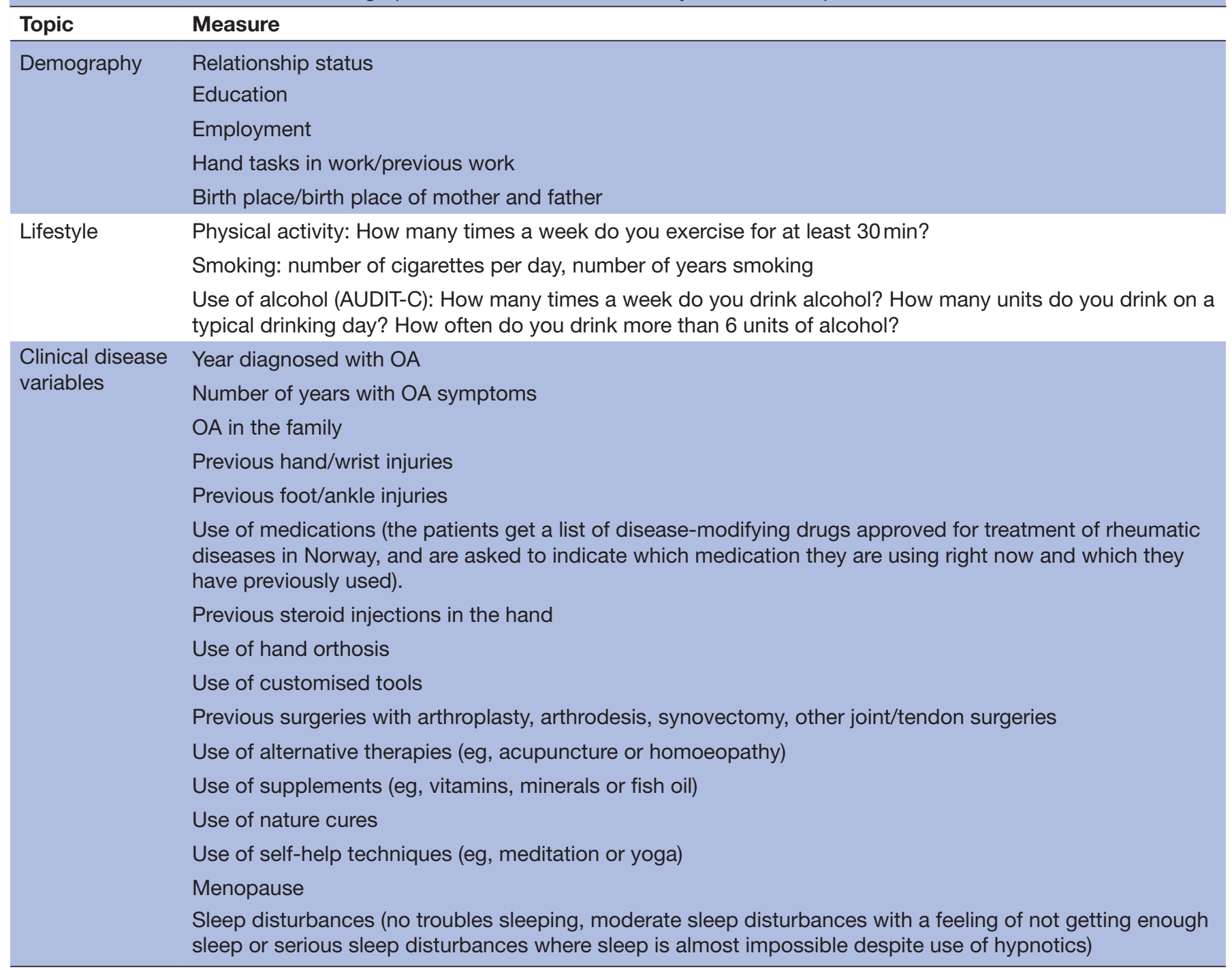

AUDIT-C, Alcohol Use Disorders Identification Test-Consumption; OA, osteoarthritis.

the questionnaire to quantify the severity of the diseases. ${ }^{47}$ Afterwards, the medical student goes over the questions together with the patient, checking that the comorbidity corresponds with the list of medications that the patients are asked to bring with them to the test evening.

A small hair sample is collected from the back of the patient's head and will be sent to an international laboratory for quantification of mean cortisol levels. The mean cortisol level will be measured to explore associations between stress exposure and pain. The amount of hair collected is equivalent to less than the width of a pencil.

\section{Joint assessment}

One rheumatologist or one rheumatology resident examine the bilateral first carpometacarpal (CMC-1), first to fifth metacarpophalangeal (MCP), first interphalangeal (IP-1) and second to fifth proximal interphalangeal (PIP) and distal interphalangeal (DIP) joints for soft tissue swelling, bony enlargement and joint tenderness according to the EULAR handbook. ${ }^{48}$ Joint tenderness is assessed using the Doyle index. ${ }^{49}$ The overall hand OA disease activity based on these examinations is summarised on an NRS from 0 to 10 . The rheumatologist/rheumatology resident perform the joint assessment only, and are blinded to all other data collected in the study.

In the foot, the tibiotalar, talonavicular, medial naviculocuneiform, intermedial naviculocuneiform, subtalar, first metatarsophalangeal (MTP) and first to fourth tarsometatarsal (TMT) joints are examined for the absence/presence of tenderness, soft tissue swelling and bony enlargement. The fifth TMT joint is not included in the examination because of challenges related to the performance of a reliable examination of the joint because of its anatomy.

Finally, the physician investigates whether the patient fulfils the American College of Rheumatology criteria for OA in hips, knees and hands. ${ }^{50-52}$ Due to the lack of radiographs of hips and knees, we will use ultrasound features instead of conventional radiographs to complete 
Table 2 Questionnaires in the case report form

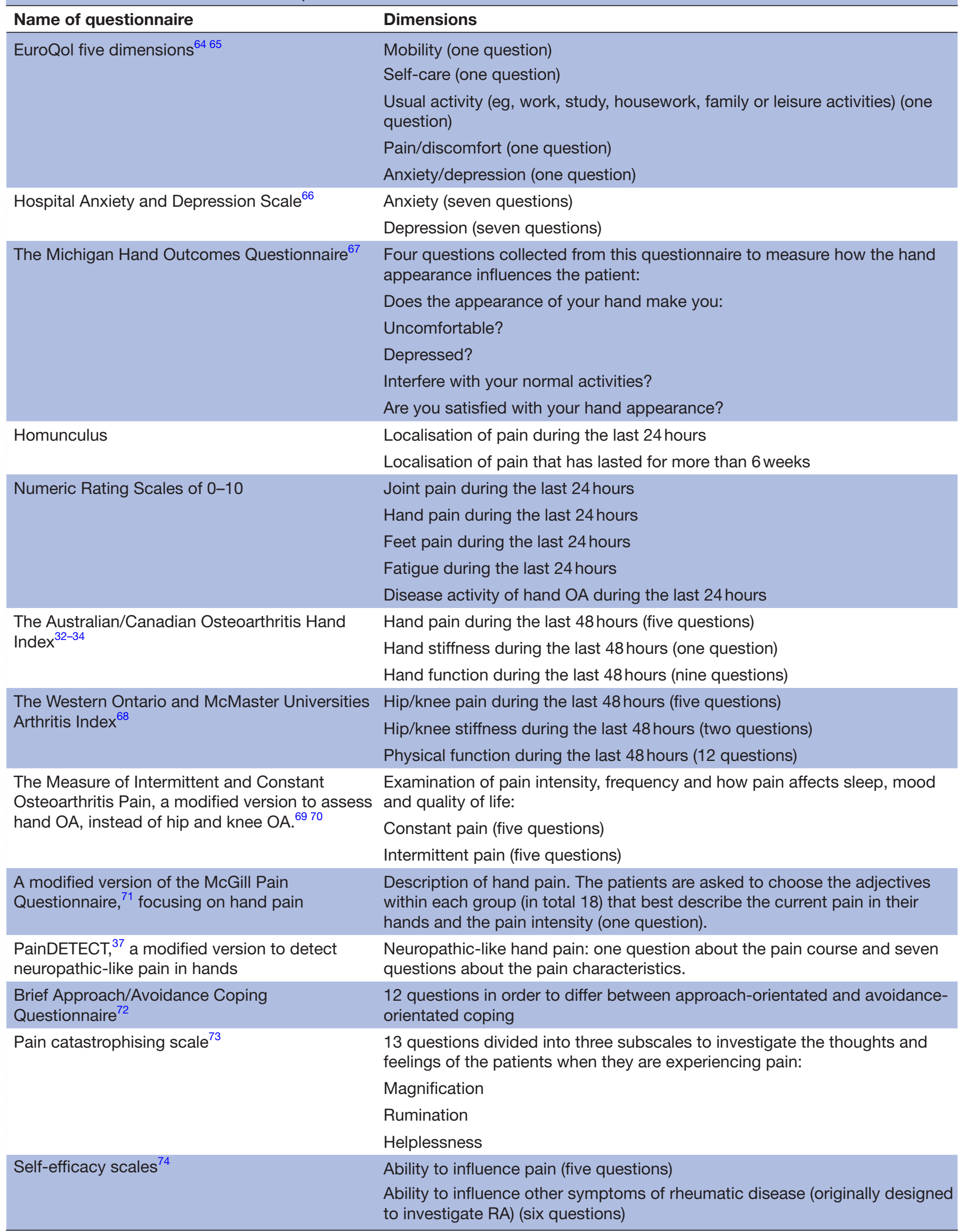


Table 2 Continued

\begin{tabular}{ll}
\hline Name of questionnaire & Dimensions \\
\hline Foot Function Index $^{75}$ & Nine questions related to pain in the feet \\
& Nine questions about disability \\
& Five questions about activity restrictions \\
& All questions are rated on Numeric Rating Scales (0-10). The questionnaire \\
& was originally developed to measure the impact of foot pain, disability and \\
& activity limitations in patients with RA. \\
\hline
\end{tabular}

OA, osteoarthritis.

the questions on imaging findings in the knee and hip criteria. Conventional radiographs of hips and knees are not collected as an attempt to reduce the total patient burden. Moreover, studies from knee and hand OA have demonstrated superior sensitivity of ultrasound to detect osteophytes as compared with conventional radiographs. ${ }^{93}$ We are not aware of any studies comparing ultrasound and conventional radiographs in hip OA.

\section{Functional tests}

Grip strength is measured by Jamar dynamometer. The procedure is completed with the patient sitting in a chair with his/her elbows at a 90-degree angle without any support of his/her arms. First, the dominant hand is tested by squeezing the dynamometer as hard as possible. This is repeated twice with $15 \mathrm{~s}$ of rest between the attempts. The measurements are noted in kilograms with one decimal precision, and then repeated for the non-dominant hand. ${ }^{54}$

The fine motor skills are tested by the Moberg Pick-up test. In total, 12 small objects are spread on a table in front of the patient within a radius of $20-30 \mathrm{~cm}$. First, the dominant hand is tested by instructing the patient to pick up the objects one-by-one as fast as possible, and place them in a small box. The procedure is then repeated with the non-dominant hand. The time from the touch of the first object to the placement of the last object in the box is measured, and the patient fails the test if he/she uses more than 300 s to pick up all the objects. ${ }^{55}$

\section{Pain sensitisation tests}

Temporal summation: A set of seven punctuate probes with fixed intensities and exerted forces of $8,16,32,64$, 128,256 and $512 \mathrm{nM}$ is used. During the investigation, the patient has his/her eyes closed, and the examiner starts with tapping the first probe against the left radioulnar joint. The patient rates the feeling of pain on the NRS from 0 to 10 , where 0 is no pain and 10 is the worst pain imaginable. The examination is repeated with the following probes in their numerical order until the patient rates the pain at 4 or higher on the NRS $(0-10)$. The probe that evoked the most pain is then tapped 10 more times with a pace of one tap per second at the same location, and the patient rates the pain of the first, fifth and tenth tap on the NRS.
Pressure pain threshold (PPT): The interphalangeal joint where the patient reports the most pain is tested with a hand-held digital algometer (FPIX25 Wagner), which is placed perpendicular to the dorsal side over the joint. The pressure of the algometer is increased with $0.5 \mathrm{~kg} / \mathrm{s}$ decided by a metronome, while the patient is resting his/her hands on a table. When the pressure becomes painful, the patient should ask for the test to be stopped. The examination is repeated three times with the algometer placed at slightly different positions over the same joint with a pause of $30 \mathrm{~s}$ between the measurements. The PPT is the average of the measurements. The patient is asked if any of his/her finger joints is pain free. The whole procedure is then repeated on this asymptomatic joint as well as on several distant control points, including the trapezius muscle, the left distal radioulnar joint and the tibialis anterior muscle.

Conditioned pain modulation: A blood pressure cuff is placed around the right upper arm of the patient approximately $3 \mathrm{~cm}$ proximal to the antecubital fossa. The blood pressure cuff is inflated to $200 \mathrm{~mm} \mathrm{Hg}$, while the patient holds a mantle of $0.5 \mathrm{~kg}$ and flexes and extends the wrist 10 times. Meanwhile, the patient rates the pain in the forearm on a $0-10$ NRS. If the patient reports pain below 4 , he/she is asked to perform another five exercises and so on until the level of pain is 4 or above. The amount of exercises completed and the pain rating in the forearm are noted. A repetition of the PPT test is performed on the left radioulnar joint when blood pressure cuff is still inflated. The procedure is stopped if the blood pressure cuff has been inflated for more than 2 min without the patient reporting pain.

Light touch: Two von Frey filaments with a strength of $2 \mathrm{~g}$ and $26 \mathrm{~g}$ are each used to touch the patient four times at the left distal radioulnar joint, which is used as a control site, and afterwards used again on the finger joint where the patient reports the most pain. The patient has his/her eyes closed, and the filaments are pressed against the skin with enough pressure to bend them. The patient is asked to report when he/she feels the filament touching the skin, and grade on the $0-10$ NRS if he/she finds it painful. Finally, the procedure is repeated with a safety pin. With every touch of the skin, the examiner ensures that the skin is only lightly touched and not penetrated by the safety pin. However, if the pin causes a slight 
bleeding, it is carefully sterilised before it is used on the next patient.

The pain sensitisation tests are selected based on what we believe is tolerable for the patients. The PPT tests may affect the microcirculation in that particular area for a few minutes afterwards, and the patients never undergo the FOI examination right after the pain tests. The patients always wait for at least $30 \mathrm{~min}$ before they undergo the FOI examination.

\section{Ultrasound}

Ultrasound of the bilateral hands, acromioclavicular joints, feet, knees and hips are performed. To ensure standardised investigations, one trained medical student performs the upper extremity examination and another medical student performs the lower extremity examination throughout the study. The medical students have been trained and supervised by experienced ultrasonographers $(\mathrm{HBH}, \mathrm{AM})$ who step in when the students are prohibited to attend. These ultrasonographers have 5-16 years of experience. Before the first test evening, the medical students were instructed in detail how to perform the ultrasound assessments. In addition, the student and the expert performed the assessments at the first three test evenings together to ensure agreement of the scoring. A reliability exercise is being performed, where the expert and the student separately and independently scores all the joint regions. The results from the reliability exercise will be presented in future papers.

A GE Logic S8 ultrasound machine is used for investigation of the upper extremity, while the lower extremity is investigated with a GE Logic E9 ultrasound machine. The same machines with fixed settings are used throughout the study to make the assessments as standardised as possible. The Doppler is optimised as previously recommended by Torp-Pedersen $e t a l,{ }^{56}$ and there are no upgrades on the machines during the study.

At the ultrasound investigation of the hands, the bilateral CMC-1, first to fifth MCP, IP-1 and the second to fifth PIP and DIP joints are longitudinally scanned from the radial to the ulnar dorsal side with the patient sitting opposite the investigator with his/her hands on a table. In addition, a transverse scanning is completed if the presence of pathology is uncertain. A scoring system for ultrasound features of hand OA made by a group of experts is used to investigate the joints. ${ }^{10}$ This scoring system includes synovial hypertrophy and/or effusion, power Doppler signals and osteophytes, all on semiquantitative scales $(0-3)$. To improve the reliability of the scoring, atlases of previously collected representative images of osteophytes and synovitis are being used. ${ }^{957}$

Ultrasound scanning of the bilateral acromioclavicular joints are performed with the patient sitting with his/her shoulders in a neutral position. Both acromioclavicular joints are investigated with the probe placed over the joints in the long axis of the clavicle. Osteophytes are scored on a $0-3$ scale, where 0 is normal, 1 is mild pathology, 2 is moderate pathology and 3 is severe pathology.
The hips are investigated with the patient lying on an examination bed in supine position with the hips and knees extended and the feet in neutral position. The anterior aspects of the proximal femur (head and neck) are evaluated for osteophytes and capsular height in a longitudinal view. Osteophytes are scored on a $0-3$ scale of occurrence, where 0 is none, 1 is mild degree, 2 is medium degree and 3 is severe degree of osteophytes. ${ }^{58}$ Capsular height is recorded as the largest perpendicular distance (in millimetres) from the middle of the femoral neck to the capsule. If fluid is present, the distance will increase.

The bilateral knees are assessed when the patient is lying in supine position on an examination bed. Osteophytes in medial and lateral tibiofemoral joints are investigated and scored on a scale from 0 to 3 with the knees in an extended position. ${ }^{53}$ In addition, synovitis/effusion in the suprapatellar recess is scored on a 0-3 scale according to a previously developed ultrasound atlas. ${ }^{57}$ The probe is placed in the sagittal plane lateral of the midline while the patient still has his/her knees fully extended (with active extension to increase fluid collection in the suprapatellar recess). Finally, the cartilage height in the femoral sulcus is measured in millimetres with the knees maximally flexed. We only assess the thickness of the cartilage in the sulcus, from the top of the interphase to the top of the bone surface. The probe is then placed transversely and just proximal to the patella.

The ultrasound investigation of the feet includes the bilateral tibiotalar, talonavicular, first to third naviculocuneiform, first to fourth TMT, first MTP, IP-1, lateral subtalar, medial subtalar and the calcaneocuboid joints. The patient is lying in supine position with flexed knee and his/her foot resting on the examination bed. The fifth TMT joint is not included because of difficulties performing a reliable assessment of this joint because of its anatomy. The presence of osteophytes, grey scale synovitis (hypertrophy and/or effusion) and power Doppler signals are scored on a $0-3$ scale. Finally, we investigate whether the spring ligament is intact.

\section{Fluorescence optical imaging}

The Xiralite scanner is used to examine the degree of disturbed microcirculation as a proxy for joint inflammation in both hands. It includes a scanner with LED lights, a computer and a camera taking pictures every second for $6 \mathrm{~min}$. The patient sets his/her hands on a preformed hand rest, and is given intravenous fluorescence dye (ICG pulsion, $0.1 \mathrm{mg} / \mathrm{kg}$ of the body weight). Patients with poor liver function (transaminases above twice the upper reference limit), poor renal function (glomerular filtration rate below $40 \mathrm{~mL} / \mathrm{min}$ ), untreated hyperthyroidism (fT4 above $21 \mathrm{pmol} / \mathrm{L}$ and thyroid-stimulating hormone (TSH) below $0.5 \mathrm{mIE} / \mathrm{L}$ ), or a known allergy to iodine or indocyanine are excluded from the FOI investigation. In addition, women who are pregnant or breastfeeding should be excluded, although it has not been relevant in the Nor-Hand study. 
During examination, enhancement of the fluorescence dye occurs in the hands, and a trained $\mathrm{PhD}$ student will later score the intensity of enhancement in the different joints on a scale from 0 to 3 . Grade 1 inflammation is red, grade 2 is intense red, while grade 3 is white on a red background. The examiner does the grading by looking at the composite image, which is derived from the first 240 images taken by the camera. In addition, an evaluation of three different phases will be performed. Phase 1 is right after the fluorescence dye is given, and studies have proposed that increased intensity in this phase represents high inflammation activity in the joint. Phase 2 is the period with fluorescence enhancement in the fingertips, and is shown to be the most sensitive to detect inflammation. In the third phase, signals from the fingertips are no longer visualised, but fluorescence signals from inflamed tissue may remain. Studies indicate that inflammation in the later phase is connected to increased capillary permeability. ${ }^{25}{ }^{26}$ A trained medical student performs the investigation, and this student is blinded to all other results. One PhD student, who has not performed the FOI examination, will later score the images according to a standardised scoring system. ${ }^{25}$

\section{Conventional radiographs}

Bilateral frontal images of the hands are obtained with a posterior-anterior view (source to image-receptor distance (SID): $115 \mathrm{~cm}$, exposure: $46 \mathrm{kVp}$ and $2 \mathrm{mAs}$ ). The patient sits with both hands pronated and the palmar surfaces placed on the detector. A slight ulnar deviation of the hands ensures that the index fingers are extensions of the long axis of radius. A trained reader will evaluate the DIP, PIP, MCP and thumb base joints according to validated scoring systems, such as the Kellgren-Lawrence scale ${ }^{5960}$ the Osteoarthritis Research Society International atlas ${ }^{61}$ and the Verbruggen Veys anatomical phase scoring system. ${ }^{62}$

Furthermore, frontal images of the bilateral feet as well as oblique and side images of each foot are obtained (SID $115 \mathrm{~cm}$, exposure: $52 \mathrm{kVp}$ and $2 \mathrm{mAs}$ ). The patient is lying on an examination bed while the knees are bent so their feet can be placed straight down on the detector. The talonavicular joint, the medial, intermedial and lateral naviculocuneiform joints, the first to fourth TMT joints and the first MTP and IP joint will be evaluated for radiographic OA features.

\section{MRI of dominant hand}

Patients are scheduled for an MRI of their dominant hand at a private imaging centre (Volvat) in Oslo, Norway. The acquisition is performed with a Siemens Aera 1.5T MRI scanner (Germany) and a 16-channel hand/wrist coil with a field of view covering both the thumb base and the interphalangeal joints. Intravenous contrast (Dotarem $279.3 \mathrm{mg} / \mathrm{mL}, 0.2 \mathrm{~mL} / \mathrm{kg}$ body weight) is given unless contraindications, for example, previous allergic reactions or reduced kidney function (glomerular filtration rate $<40 \mathrm{~mL} / \mathrm{min}$ ). The patients are supinated with feet first and the dominant hand along their side. The details of the sequences are shown in table 3. The T1 volumetric interpolated breath-hold examination (VIBE) with water excitation sequences are reconstructed into axial, coronal and sagittal planes with $2 \mathrm{~mm}$ slice thickness. The axial planes of the carpus were defined according to carpal bones, whereas the axial planes of the fingers were perpendicular to the metacarpal bones in the coronal plane. The sagittal slices were perpendicular to the coronal slices. The MRIs will be scored according to validated scoring systems for the interphalangeal joints (Hand OA MRI scoring system; HOAMRIS $)^{13} 63$ and thumb base joints (Thumb base OA MRI scoring system; TOMS). ${ }^{14}$ The MRIs will be read by future PhD students, supervised by a musculoskeletal radiologist (KF) and an experienced reader $(\mathrm{IKH})$.

\section{CT of dominant hand}

CT is performed of the dominant hand using a 64-channel 750 HD Discovery Machine (General Electric, USA) (exposure: $120 \mathrm{kVp}, 30 \mathrm{mAs}$ ). The patients are placed in 'Superman' position with the dominant arm straightened above their head and the non-dominant hand along their body. This position prevents unnecessary radiation of the head and internal organs. The radiation dose, that is, CT Dose Index Volume is 4,3 mGy. Scanning is performed with $0.625 \mathrm{~mm}$ thin slices, which are automatically reconstructed to $1 \mathrm{~mm}$ thick slices in axial, coronal and sagittal planes. Because there are no available hand OA scoring systems for CT scans, we must define the features

Table 3 Details of MRI sequences

Coronal T1 SE Coronal PD Dixon Axial PD TSE fs Sagittal T1 TSE $\begin{aligned} & \text { Coronal T1 Vibe W } \\ & \text { and after contrast }\end{aligned}$

\begin{tabular}{llllll}
\hline TE $(\mathrm{ms})$ & 11 & 31 & 26 & 11 & 6.94 \\
TR $(\mathrm{ms})$ & 549 & 3970 & 3630 & 509 & 17 \\
$\begin{array}{l}\text { Slice } \\
\text { thickness }(\mathrm{mm})\end{array}$ & 2.5 & 2.5 & 3.2 & 3 & 0.4 \\
\hline $\begin{array}{l}\text { Spacing }(\mathrm{mm}) \\
\text { Matrix }\end{array}$ & 0.2 & 0.2 & 0.6 & 0.3 & 0 \\
\hline
\end{tabular}

fs, fat saturation; PD, proton density; SE, spin echo; TE, echotime; TR, repetition time; TSE, turbo spin echo; WE, water excitation. 
and their grading before we start the scoring of images. This work will be based on previously developed scoring systems for MRI and conventional radiographs. The CT scans will be read by a future $\mathrm{PhD}$ student under supervision of a musculoskeletal radiologist (KF) and the project leader (IKH).

\section{Blood tests/Biobank}

In order to check the eligibility criteria, blood samples are collected to measure ESR, $\mathrm{C}$ reactive protein, ferritin, anti-CCP and rheumatoid factor at the screening examination. In addition, the glomerular filtration rate is measured at a maximum of 3 months before the contrast-enhanced MRI. Alanine amino transferase (ALAT), aspartate amino transferase (ASAT) and thyroid status with fT 4 and TSH are measured before the FOI scanning. At the test evening, whole blood, plasma and serum, in addition to a urine sample, are collected and stored in a certified biobank, which consists of a freezer with a temperature of $-70^{\circ} \mathrm{C}$. These samples will later be used only for research.

\section{Project timeline}

April 2016-May 2017: Carry out the baseline examination. 2017-2020: Data analysis and submission of results from the baseline examination.

2020: Carry out the first follow-up examination.

2020-2023: Data analysis and submission of results from the first follow-up examination.

2024: Carry out the second follow-up examination.

2024-2027: Data analysis and submission of results from the second follow-up examination.

\section{Statistical analysis}

We will perform both parametric and non-parametric statistical analyses, relying on the distribution of variables. We plan to perform cross-sectional analyses when all participants have finished the baseline examination, and longitudinal analyses when participants have finished at least one of the two planned follow-up examinations. Reliability will be evaluated using, for example, kappa values and intraclass correlation coefficients. Agreement and associations between different relevant biomarkers and associations to patient-reported outcomes will be explored by, for example, regression analyses of our cross-sectional data. Analyses will be performed at patient level as well as joint level depending on our research question, and the dependency between joints within one patient will be taken into account. The selection of independent and dependent variables in our analyses will depend on the research question. Our analyses will be adjusted for multiple comparisons, if appropriate. Different hand OA subgroups will be compared. In longitudinal analyses, we will evaluate the predictive value of the baseline variables on hand OA outcomes as well as the sensitivity to change and the inter-relationship between OA biomarkers and patient-reported outcomes.

\section{ETHICS AND DISSEMINATION}

This project is approved by the Norwegian Regional Committee for Medical and Health Research Ethics (Ref. no: 2014/2057). The approval application was submitted in October 2014, and the approval was given in February 2015. A change notice due to inclusion of more imaging modalities in the study was submitted in September 2015, which was approved in October 2015. The study is registered at https://clinicaltrials.gov (Ref. no: NCT03083548).

The participants receive oral and written information about the project in advance of the test evening. Additionally, they receive oral information about the different investigations at the test evening. It is made clear to the patients that participation in the study is voluntary. A consent form is signed before participation, and the participants can whenever they want withdraw their consent without further explanation. All data obtained in the study will be de-identified and safely stored on the research server at Diakonhjemmet Hospital. It will not be possible to relate the collected data to a specific participant without a code list, which is kept separate from the file with the collected data.

The study findings will be analysed and submitted to peer-reviewed international rheumatology journals. The data analysis will start after the baseline examination is completed in May 2017, and the results will be presented at international and national congresses and in peer-reviewed international journals from 2018. Additionally, more papers will derive from the follow-up examinations.

\section{DISCUSSION}

Limited research has been performed on hand $\mathrm{OA}$ in contrast to, for example, knee OA. More research is needed on the natural disease course, and there is therefore a need for observational cohorts of patients with hand OA.

In the Nor-Hand study, we have included a broad range of pain questionnaires that are not commonly used in hand OA research in order to better characterise the pain in these patients. We believe this will provide additional information about the pain characteristics, which can be relevant to understand the pain mechanisms in hand OA. Furthermore, as the first large-scale study, our patients undergo an examination of pain sensitisation, providing additional information about peripheral and central sensitisation. Our results may have direct implications for pain management of the patients.

Additionally, one of our major aims in the Nor-Hand study is to validate different imaging outcome measures to be used in future clinical trials. Moreover, the imaging data will be explored in association to the pain data, giving us increased knowledge about the role of structural and inflammatory features as causes of pain in hand OA. To our knowledge, no previous hand OA study has included conventional radiographs, ultrasound, MRI, CT and FOI in their study protocol. The Nor-Hand study will be the 
first large-scale study on FOI findings in hand OA. Evaluation of the FOI images may provide additional information about the inflammatory characteristics in hand $\mathrm{OA}$, which is not covered by MRI and ultrasound.

The generalisability of the Nor-Hand study is limited due to its hospital-based study design, as most patients with hand $\mathrm{OA}$ are being managed in a primary care setting. However, we aim for an inclusion of patients with a broad range of symptoms, and patients with both early and severe hand OA are being included. In order to avoid patients with a systemic inflammatory joint disease, we exclude patients with elevated inflammatory markers, rheumatoid factor and anti-CCP positivity, as well as a diagnosis of psoriasis. Persons with elevated ferritin take additional blood tests to evaluate the iron saturation, in order to exclude persons with haemochromatosis. Iron saturation up to $50 \%$ is accepted. Hence, we believe that the Nor-Hand study will consist of patients with primary hand OA only, although we can not rule out that our patients will develop another joint disease later in life.

To ensure standardised investigations, we aim to have the same trained examiner performing one specific examination throughout the whole study. In addition, each examiner is blinded to the results from the other examinations.

Furthermore, we try to minimise the amount of missing data. All patients will complete the questionnaires and the physical examination at the test evening, in addition to conventional radiographs after the test evening. However, patients might be unwilling to undergo the FOI, CT and MRI due to concerns about, for example, contrast agents, fluorescence dye or radiation. The final amount of missing data for the MRI is estimated to be less than $10 \%$, but not all patients have contrast-enhanced imaging. The missing data of the FOI investigation are estimated somewhat higher (less than 20\%), which is related to patients' concern about possible allergic reactions to the fluorescence dye, or difficulties with insertion of the peripheral venous cannula required for injection of the fluorescence dye. It should be noted that no allergic reactions have occurred during the study.

The Nor-Hand study will provide innovative knowledge about the natural disease course of hand OA, including increased information about pain characteristics, and validation of imaging biomarkers. We believe our results will be of importance for performance of future clinical trials, in addition to clinical management of patients with hand OA.

Contributors MG: acquisition of data, drafting the article and final approval of the manuscript.EM: acquisition of data (project coordinator), critical revision and final approval of the manuscript. AM, HBH: study design (ultrasound), critical revision and final approval of the manuscript. BSC: acquisition of data, critical revision and final approval of the manuscript. KF: study design (imaging), critical revision and final approval of the manuscript. TI, KM: study design (MRI), critical revision and final approval of the manuscript. TN: study design (pain), critical revision and final approval of the manuscript. TKK: study design, critical revision and final approval of the manuscript. IKH: study design, drafting the article, critical revision and final approval of the manuscript.
Funding The data collection of the Nor-Hand study is supported by grants from the South Eastern Norway Regional Health Authority, Simon Fougner Hartmanns Family foundation, Trygve Gythfeldt's research foundation and Pahles foundation.

Competing interests None declared.

Patient consent Obtained.

Ethics approval Norwegian Regional Committee for Medical and Health Research Ethics.

Provenance and peer review Not commissioned; externally peer reviewed.

Open Access This is an Open Access article distributed in accordance with the Creative Commons Attribution Non Commercial (CC BY-NC 4.0) license, which permits others to distribute, remix, adapt, build upon this work non-commercially, and license their derivative works on different terms, provided the original work is properly cited and the use is non-commercial. See: http://creativecommons.org/ licenses/by-nc/4.0/

(c) Article author(s) (or their employer(s) unless otherwise stated in the text of the article) 2017. All rights reserved. No commercial use is permitted unless otherwise expressly granted.

\section{REFERENCES}

1. Lawrence RC, Felson DT, Helmick CG, et al. Estimates of the prevalence of arthritis and other rheumatic conditions in the United States. Part II. Arthritis Rheum 2008;58:26-35.

2. Slatkowsky-Christensen B, Mowinckel P, Loge JH, et al. Healthrelated quality of life in women with symptomatic hand osteoarthritis: a comparison with rheumatoid arthritis patients, healthy controls, and normative data. Arthritis Rheum 2007;57:1404-9.

3. Kloppenburg M, Kwok WY. Hand osteoarthritis-a heterogeneous disorder. Nat Rev Rheumatol 2011;8:22-31.

4. Haugen IK, Englund M, Aliabadi P, et al. Prevalence, incidence and progression of hand osteoarthritis in the general population: the Framingham Osteoarthritis Study. Ann Rheum Dis 2011;70:1581-6.

5. Haugen IK, Mathiessen A, Slatkowsky-Christensen B, et al. Synovitis and radiographic progression in non-erosive and erosive hand osteoarthritis: is erosive hand osteoarthritis a separate inflammatory phenotype? Osteoarthritis Cartilage 2016;24:647-54.

6. Bijsterbosch J, Visser W, Kroon HM, et al. Thumb base involvement in symptomatic hand osteoarthritis is associated with more pain and functional disability. Ann Rheum Dis 2010;69:585-7.

7. Zhang W, Doherty M, Leeb BF, et al. EULAR evidence-based recommendations for the diagnosis of hand osteoarthritis: report of a task force of ESCISIT. Ann Rheum Dis 2009;68:8-17.

8. Kloppenburg M, Bøyesen P, Visser AW, et al. Report from the OMERACT Hand Osteoarthritis Working Group: set of core domains and preliminary set of instruments for use in clinical trials and observational studies. J Rheumatol 2015;42:2190-7.

9. Mathiessen A, Haugen IK, Slatkowsky-Christensen B, et al. Ultrasonographic assessment of osteophytes in 127 patients with hand osteoarthritis: exploring reliability and associations with MRI, radiographs and clinical joint findings. Ann Rheum Dis 2013;72:51-6.

10. Keen HI, Lavie F, Wakefield RJ, et al. The development of a preliminary ultrasonographic scoring system for features of hand osteoarthritis. Ann Rheum Dis 2008;67:651-5.

11. Guermazi A, Roemer FW, Hayashi D. Imaging of osteoarthritis: update from a radiological perspective. Curr Opin Rheumatol 2011;23:484-91.

12. Haugen IK, Lillegraven S, Slatkowsky-Christensen B, et al. Hand osteoarthritis and MRI: development and first validation step of the proposed Oslo Hand Osteoarthritis MRI score. Ann Rheum Dis 2011;70:1033-8.

13. Haugen IK, Østergaard M, Eshed I, et al. Iterative development and reliability of the OMERACT hand osteoarthritis MRI scoring system. J Rheumatol 2014;41:386-91.

14. Kroon FP, Conaghan PG, Foltz V, et al. Development and Reliability of the OMERACT thumb base osteoarthritis magnetic resonance imaging scoring system. J Rheumatol 2017. [Epub ahead of print: 01 Mar 2017].

15. Haugen IK, Bøyesen P, Slatkowsky-Christensen B, et al. Comparison of features by MRI and radiographs of the interphalangeal finger joints in patients with hand osteoarthritis. Ann Rheum Dis 2012;71:345-50.

16. Haugen IK, Slatkowsky-Christensen B, Faraj K, et al. The comparison of magnetic resonance imaging and radiographs to assess structural progression over 5 years in hand osteoarthritis. Rheumatology 2017;56:kew419. 
17. Haugen IK, Slatkowsky-Christensen B, Bøyesen P, et al. MRI findings predict radiographic progression and development of erosions in hand osteoarthritis. Ann Rheum Dis 2016;75:117-23.

18. Damman W, Liu R, Bloem JL, et al. Bone marrow lesions and synovitis on $\mathrm{MRI}$ associate with radiographic progression after 2 years in hand osteoarthritis. Ann Rheum Dis 2017;76:214-7.

19. Mathiessen A, Slatkowsky-Christensen B, Kvien TK, et al. Ultrasound-detected inflammation predicts radiographic progression in hand osteoarthritis after 5 years. Ann Rheum Dis 2016;75:825-30.

20. Kortekaas MC, Kwok WY, Reijnierse M, et al. Inflammatory ultrasound features show independent associations with progression of structural damage after over 2 years of follow-up in patients with hand osteoarthritis. Ann Rheum Dis 2015;74:1720-4.

21. Haugen IK, Slatkowsky Christensen B, Bøyesen P, et al. Increasing synovitis and bone marrow lesions are associated with incident joint tenderness in hand osteoarthritis. Ann Rheum Dis 2016;75:702-8.

22. Kortekaas MC, Kwok WY, Reijnierse M, et al. Pain in hand osteoarthritis is associated with inflammation: the value of ultrasound. Ann Rheum Dis 2010;69:1367-9.

23. Haugen IK, Bøyesen P, Slatkowsky-Christensen B, et al. Associations between MRI-defined synovitis, bone marrow lesions and structural features and measures of pain and physical function in hand osteoarthritis. Ann Rheum Dis 2012:71:899-904.

24. Verbruggen G, Wittoek R, Vander Cruyssen B, et al. Tumour necrosis factor blockade for the treatment of erosive osteoarthritis of the interphalangeal finger joints: a double blind, randomised trial on structure modification. Ann Rheum Dis 2012;71:891-8.

25. Werner SG, Langer HE, Ohrndorf S, et al. Inflammation assessment in patients with arthritis using a novel in vivo fluorescence optical imaging technology. Ann Rheum Dis 2012;71:504-10.

26. Werner SG, Langer HE, Schott $P$, et al. Indocyanine green-enhanced fluorescence optical imaging in patients with early and very early arthritis: a comparative study with magnetic resonance imaging. Arthritis Rheum 2013:65:3036-44.

27. Meier R, Thürmel K, Moog P, et al. Detection of synovitis in the hands of patients with rheumatologic disorders: diagnostic performance of optical imaging in comparison with magnetic resonance imaging. Arthritis Rheum 2012;64:2489-98.

28. Glimm AM, Werner SG, Burmester GR, et al. Analysis of distribution and severity of inflammation in patients with osteoarthitis compared to rheumatoid arthritis by ICG-enhanced fluorescence optical imaging and musculoskeletal ultrasound: a pilot study. Ann Rheum Dis 2016;75:566-70.

29. Saltzherr MS, Selles RW, Bierma-Zeinstra SM, et al. Metric properties of advanced imaging methods in osteoarthritis of the hand: a systematic review. Ann Rheum Dis 2014;73:365-75.

30. Hawker GA, Stewart L, French MR, et al. Understanding the pain experience in hip and knee osteoarthritis-an OARSI/OMERACT initiative. Osteoarthritis Cartilage 2008;16:415-22.

31. Neogi T. The epidemiology and impact of pain in osteoarthritis. Osteoarthritis Cartilage 2013;21:1145-53.

32. Slatkowsky-Christensen B, Kvien TK, Bellamy N. Performance of the Norwegian version of AUSCAN-a disease-specific measure of hand osteoarthritis. Osteoarthritis Cartilage 2005;13:561-7.

33. Bellamy N, Campbell J, Haraoui B, et al. Clinimetric properties of the AUSCAN Osteoarthritis Hand Index: an evaluation of reliability, validity and responsiveness. Osteoarthritis Cartilage 2002;10:863-9.

34. Bellamy N, Campbell J, Haraoui B, et al. Dimensionality and clinical importance of pain and disability in hand osteoarthritis: development of the Australian/Canadian (AUSCAN) osteoarthritis hand index. Osteoarthritis Cartilage 2002;10:855-62.

35. Felson DT. The sources of pain in knee osteoarthritis. Curr Opin Rheumatol 2005;17:624-8.

36. Kidd BL. Osteoarthritis and joint pain. Pain 2006;123:6-9.

37. Freynhagen $R$, Baron $R$, Gockel U, et al. painDETECT: a new screening questionnaire to identify neuropathic components in patients with back pain. Curr Med Res Opin 2006;22:1911-20.

38. Hochman JR, Davis AM, Elkayam J, et al. Neuropathic pain symptoms on the modified painDETECT correlate with signs of central sensitization in knee osteoarthritis. Osteoarthritis Cartilage 2013;21:1236-42.

39. Imamura M, Imamura ST, Kaziyama HH, et al. Impact of nervous system hyperalgesia on pain, disability, and quality of life in patients with knee osteoarthritis: a controlled analysis. Arthritis Rheum 2008;59:1424-31.

40. Lee YC, Lu B, Bathon JM, et al. Pain sensitivity and pain reactivity in osteoarthritis. Arthritis Care Res 2011;63:320-7.

41. Arendt-Nielsen L, Nie H, Laursen MB, et al. Sensitization in patients with painful knee osteoarthritis. Pain 2010;149:573-81.
42. Wajed J, Ejindu V, Heron C, et al. Quantitative sensory testing in painful hand osteoarthritis demonstrates features of peripheral sensitisation. Int J Rheumatol 2012;2012:1-8.

43. Chiarotto A, Fernandez-de-Las-Peñas $C$, Castaldo $M$, et al. Widespread pressure pain hypersensitivity in elderly subjects with unilateral thumb carpometacarpal osteoarthritis. Hand 2013;8:422-9.

44. Chiarotto A, Fernandez-de-Las-Peñas C, Castaldo M, et al. Bilateral pressure pain hypersensitivity over the hand as potential sign of sensitization mechanisms in individuals with thumb carpometacarpal osteoarthritis. Pain Med 2013;14:1585-92.

45. Sofat N, Smee C, Hermansson M, et al. Functional MRI demonstrates pain perception in hand osteoarthritis has features of central pain processing. J Biomed Graph Comput 2013;3.

46. Riyazi N, Meulenbelt I, Kroon HM, et al. Evidence for familial aggregation of hand, hip, and spine but not knee osteoarthritis in siblings with multiple joint involvement: the GARP study. Ann Rheum Dis 2005;64:438-43.

47. Sangha O, Stucki G, Liang MH, et al. The self-administered comorbidity questionnaire: a new method to assess comorbidity for clinical and health services research. Arthritis Rheum 2003;49:156-63.

48. van Riel PLCM L, Scott D. Eular handbook of clinical assessments in rheumatoid arthritis: Van Zuiden communications BV, Alphen a/d Rijn 2004, 2004.

49. Bijsterbosch J, Wassenaar MJ, le Cessie S, et al. Doyle index is a valuable additional pain measure in osteoarthritis. Osteoarthritis Cartilage 2010;18:1046-50.

50. Altman R, Alarcón G, Appelrouth D, et al. The American College of Rheumatology criteria for the classification and reporting of osteoarthritis of the hand. Arthritis Rheum 1990;33:1601-10.

51. Altman R, Asch E, Bloch D, et al. Development of criteria for the classification and reporting of osteoarthritis. Classification of osteoarthritis of the knee. Diagnostic and Therapeutic Criteria Committee of the American Rheumatism Association. Arthritis Rheum 1986;29:1039-49.

52. Altman R, Alarcón G, Appelrouth D, et al. The American College of Rheumatology criteria for the classification and reporting of osteoarthritis of the hip. Arthritis Rheum 1991;34:505-14.

53. Koski JM, Kamel A, Waris P, et al. Atlas-based knee osteophyte assessment with ultrasonography and radiography: relationship to arthroscopic degeneration of articular cartilage. Scand J Rheumatol 2016;45:158-64.

54. Schmidt RT, Toews JV. Grip strength as measured by the Jamar dynamometer. Arch Phys Med Rehabil 1970;51:321-7.

55. Ng CL, Ho DD, Chow SP. The Moberg pickup test: results of testing with a standard protocol. J Hand Ther 1999;12:309-12.

56. Torp-Pedersen S, Christensen R, Szkudlarek M, et al. Power and color Doppler ultrasound settings for inflammatory flow: impact on scoring of disease activity in patients with rheumatoid arthritis. Arthritis Rheumatol 2015;67:386-95.

57. Hammer HB, Bolton-King P, Bakkeheim V, et al. Examination of intra and interrater reliability with a new ultrasonographic reference atlas for scoring of synovitis in patients with rheumatoid arthritis. Ann Rheum Dis 2011;70:1995-8.

58. Qvistgaard E, Torp-Pedersen S, Christensen R, et al. Reproducibility and inter-reader agreement of a scoring system for ultrasound evaluation of hip osteoarthritis. Ann Rheum Dis 2006;65:1613-9.

59. Kellgren JH, Lawrence JS. Radiological assessment of osteoarthrosis. Ann Rheum Dis 1957:16:494-502.

60. Marshall M, Dziedzic KS, van der Windt DA, et al. A systematic search and narrative review of radiographic definitions of hand osteoarthritis in population-based studies. Osteoarthritis Cartilage 2008;16:219-26.

61. Altman RD, Gold GE. Atlas of individual radiographic features in osteoarthritis, revised. Osteoarthritis Cartilage 2007;15(Suppl A):A1-56.

62. Verbruggen G, Veys EM. Numerical scoring systems for the progression of osteoarthritis of the finger joints. Rev Rhum Engl Ed 1995;62:27s-32.

63. Haugen IK, Eshed I, Gandjbakhch F, et al. The longitudinal reliability and responsiveness of the OMERACT Hand Osteoarthritis Magnetic Resonance Imaging Scoring System (HOAMRIS). J Rheumatol 2015;42:2486-91.

64. EuroQol Group. EuroQol-a new facility for the measurement of health-related quality of life. Health Policy 1990;16:199-208.

65. Räsänen $P$, Roine $E$, Sintonen $\mathrm{H}$, et al. Use of quality-adjusted life years for the estimation of effectiveness of health care: A systematic literature review. Int J Technol Assess Health Care 2006;22:235-41.

66. Zigmond AS, Snaith RP. The hospital anxiety and depression scale. Acta Psychiatr Scand 1983;67:361-70. 
67. Chung KC, Pillsbury MS, Walters MR, et al. Reliability and validity testing of the michigan hand outcomes questionnaire. $J$ Hand Surg Am 1998;23:575-87.

68. Bellamy N, Buchanan WW, Goldsmith $\mathrm{CH}$, et al. Validation study of WOMAC: a health status instrument for measuring clinically important patient relevant outcomes to antirheumatic drug therapy in patients with osteoarthritis of the hip or knee. $J$ Rheumatol 1988;15:1833-40.

69. Hawker GA, Davis AM, French MR, et al. Development and preliminary psychometric testing of a new OA pain measure-an OARSI/OMERACT initiative. Osteoarthritis Cartilage 2008;16:409-14.

70. Maillefert JF, Kloppenburg M, Fernandes L, et al. Multi-language translation and cross-cultural adaptation of the OARSI/OMERACT measure of intermittent and constant osteoarthritis pain (ICOAP). Osteoarthritis Cartilage 2009;17:1293-6.
71. Melzack R. The McGill Pain Questionnaire: major properties and scoring methods. Pain 1975;1:277-99.

72. Finset A, Steine S, Haugli L, et al. The Brief Approach/Avoidance Coping Questionnaire: development and validation. Psychol Health Med 2002;7:75-85.

73. Fernandes L, Storheim K, Lochting I, et al. Cross-cultural adaptation and validation of the Norwegian pain catastrophizing scale in patients with low back pain. BMC Musculoskelet Disord 2012;13:111.

74. Lorig K, Chastain RL, Ung E, et al. Development and evaluation of a scale to measure perceived self-efficacy in people with arthritis. Arthritis Rheum 1989;32:37-44.

75. Budiman-Mak E, Conrad KJ, Roach KE. The Foot Function Index: a measure of foot pain and disability. $J$ Clin Epidemiol 1991;44:561-70. 